\title{
A compact two-wave dichrometer of an optical biosensor analytical system for medicine
}

\author{
D.P. Chulkov ${ }^{1}$, V.M. Gusev ${ }^{1}$, O.N. Kompanets ${ }^{1, *}$, F.V. Vereschagin ${ }^{1}$, S.G. Skuridin ${ }^{2}$, and \\ Yu.M. Yevdokimov ${ }^{2}$ \\ ${ }^{1}$ Institute for Spectroscopy RAS, 108840 Troitsk, Moscow, Russia \\ ${ }^{2}$ Engelgardt Institute of Molecular Biology RAS, 119991 Moscow, Russia
}

\begin{abstract}
An experimental model has been developed of a compact twowave dichrometer on the base of LEDs that is well-suited to work with "liquid" DNA nanoconstructions as biosensing units. The mobile and inexpensive device is intended for use in a biosensor analytical system for rapid determination of biologically active compounds in liquids to solve practical problems of clinic medicine and pharmacology.
\end{abstract}

We develop optical biosensor analytical systems, in which "liquid" particles of the cholesteric liquid-crystalline dispersion (CLCD) of DNA are used as a biosensing unit, and a portable dichrometer registers changes of the circular dichroism (CD) signal generated with the DNA biosensing unit during its interaction with a defined biologically active compound (BAC) [1]. DNA biosensing units exhibit anomalously large optical activity in the CD spectrum in an absorption band of DNA nitrogen bases (chromophores) at $270 \mathrm{~nm}$, the magnitude of which is stable and acts as a convenient "quality" criterion of prepared DNA CLCD particles. Intercalation of coloured BACs into the DNA cholesteric liquidcrystalline structure leads to the appearance in the $\mathrm{CD}$ spectrum of an additional abnormal band in the visible spectrum, whose amplitude depends on the concentration of BAC - this calibration dependence is used then to establish the presence of such compounds in the assay medium and to determine their concentration.

Among our developments there are dichrometers with tunable wavelength light sources $[2,3]$ which, due to their versatility, are primarily used for research purposes. Taking into account the specificity of the CD spectrum generated by biosensing units based on "liquid" DNA CLCD particles at intercalation therein of coloured BAC, the idea has occured to develop a compact biosensor analytical system for the rapid detection in clinical conditions of several important BAC, in particular, antibiotics (daunorubicin and analogues) used in oncological practice. Such the biosensor would work on only two discrete wavelengths, one of which corresponds to the absorption band of DNA chromophores at a wavelength of 270 $\mathrm{nm}$, and the other - to the abnormal band in the visible region of the CD spectrum of the (DNA + BAC) complex. Such a choice predetermined steps to simplification of the dichrometer - a rejection of expensive broadband lamp (Xe) of the radiation source, a monochromator and a photomultiplier. The use of modern high power light-emitting diodes as light sources and a photodiode for registering the CD signal allows both DNA CLCD particles quality control at a wavelength of $270 \mathrm{~nm}$ and registration of the smallest changes

\footnotetext{
*Corresponding author: onkomp@isan.troitsk.ru
} 
of the abnormal $\mathrm{CD}$ signal in the visible region resulting of interaction of the detectable BAC molecules with DNA CLCD particles with formation of the (DNA + BAC) complex.

The experimental two-wave dichrometer model that is intended to determine the daunomycin (DAU), for example, uses high brightness light-emitting diodes UVTOP270TO39BL (Sensor Electronic Technology Company) operating at $270 \mathrm{~nm}$, and two LEDs (Prolight Company): the main one - S12LG2C-B emitting green light at $510 \mathrm{~nm}$ corresponding to the abnormal band in the CD spectrum of the (DNA + DAU) complex, and the auxiliary one - S12LR2C-B emitting red light at $634 \mathrm{~nm}$ and serving for fixing the "CD basic line". Radiation of LEDs is aligned along the same path by dichroic mirrors set at an angle of $45^{\circ}$ to the directions of the emitters light flows, all sources are turned on alternately. To compensate the divergence of the radiation collimating lenses are used, and for narrowing the emission spectrum to 3-6 $\mathrm{nm}$ interference filters are applied.

In addition to the LED light sources and optics to them, the two-wave dichrometer model uses the standard for the previous multifunction dichrometers [2] elements: a linear polarizer, a photoelastic modulator of circular polarization of radiation, and a temperature controlled $\left(0.5{ }^{\circ} \mathrm{C}\right.$ accuracy within the temperature range of $\left.20-80{ }^{\circ} \mathrm{C}\right)$ cuvette compartment for placement in a quartz spectrophotometric cuvette $(10 \times 10 \mathrm{~mm})$ of test samples - "liquid" biosensing unit based on DNA CLCD particles in contact with the test liquid containing the detectable BAC. To register the anomalous CD signal and its changes the photodiode S1337-1010BQ (Hamamatsu Company) is applied.

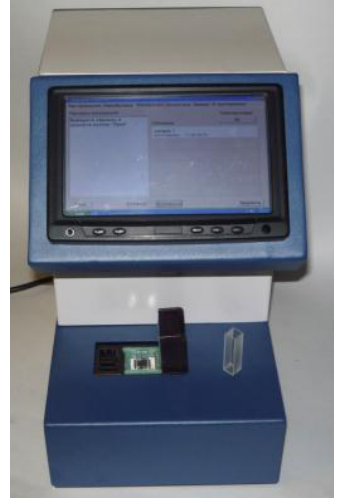

Fig. Photo of the compact experimental two-wave dichrometer model adapted to work as a part of the DNA based biosensor analytical system

The two-wave dichrometer model is implemented on the basis of a built-in computer. The motherboard VIA EPIA PX ("VIA Technologies" Company) of form factor Pico-ITX which is a full-featured motherboard for x86 processors combines multiple units of the control system. A seven-inch widescreen color LCD VGA monitor with touch screen is used to display graphic information and provide the user interface. The device has broad communication capabilities: depending on the user requirements it can transfer data over wireless channels, Wi-Fi and Bluetooth, as well as an Ethernet network.

The two-wave dichrometer model dimensions have been reduced to $210 \times 314 \times 350 \mathrm{~mm}$, its full power consumption does not exceed 100 Watts. The developed two-wave dichrometer model, being relatively compact, without any loss of sensitivity of the $\mathrm{CD}$ signal registration can be adapted to work in the biosensor test system in five modes: calibration of the system in the UV and visible regions of the spectrum; testing of optical properties of $\mathrm{BAC}$; calibration of the optical properties of biosensing units based on DNA CLCD particles, and control of their optical activity prior to use; study of the dynamics of DNA nanoconstructions transformation during their interaction with $\mathrm{BAC}$; determining the presence and concentration of BAC in the test fluid.

In the last regime mode the dichrometer operates by means of the program "Analytics" which, through a dialogue, "leads" the user through all steps prescribed with the laboratory technique of measuring a BAC concentration in the test liquid by circular dichroism (developed by the EIMB), and outputs the final result value as the BAC concentration.

The support from the Program "Basic researches for the developing biomedical technologies" of the Presidium of RAS is acknowledged.

\section{References}

1. Yu.M. Yevdokimov, V.I. Salyanov, S.G. Skuridin, Nanostructures and nanoconstructions based on DNA (CRC Press, New York, 2012)

2. O.N. Kompanets, Yu.M. Yevdokimov, Physics Uspekhi 52, 304 (2009)

3. Yu.M. Yevdokimov, O.N. Kompanets, Physics Uspekhi 57, 615 (2014) 